\title{
A point-of-care neutrophil elastase activity assay identifies bronchiectasis severity, airway infection and risk of exacerbation
}

\author{
Amelia Shoemark ${ }^{1}$, Erin Cant ${ }^{1}$, Luis Carreto $^{2}$, Alexandria Smith $^{3}$, \\ Martina Oriano ${ }^{4}$, Holly R. Keir ${ }^{1}$, Lidia Perea ${ }^{5}$, Elisabet Canto ${ }^{5}$, \\ Leonardo Terranova ${ }^{4}$, Silvia Vidal ${ }^{5}$, Kelly Moffitt ${ }^{6}$, Stefano Aliberti $\mathbb{1}^{4}$, \\ Oriol Sibila $\mathbb{1}^{5}$ and James D. Chalmers ${ }^{1}$
}

Affiliations: 'Scottish Centre for Respiratory Research, University of Dundee, Ninewells Hospital and Medical School, Dundee, UK. ${ }^{2}$ Hospital Professor Fernando Fonseca (HFF), Lisbon, Portugal. ${ }^{3}$ University of Cambridge, Cambridge, UK. ${ }^{4}$ Dept of Pathophysiology and Transplantation, University of Milan and Fondazione IRCCS Ca'Granda Ospedale Maggiore Policlinico, Respiratory Unit and Cystic Fibrosis Adult Center, Milan, Italy. ${ }^{5}$ Hospital de la Santa Creu I Sant Pau, Biomedical Research Institute (IIB-Sant Pau) Barcelona, Barcelona, Spain. ${ }^{6}$ Proaxsis Ltd, Belfast, UK.

Correspondence: James D. Chalmers, Division of Molecular and Clinical Medicine, University of Dundee, Dundee, DD1 9SY, UK. E-mail: jchalmersQdundee.ac.uk

@ERSpublications

NEATstik $^{\circ}$, a simple point-of-care semi-quantitative neutrophil elastase assay, can identify bronchiectasis patients with airway infection and patients at high risk of exacerbations over the subsequent 12 months http://bit.ly/2HL6OyK

Cite this article as: Shoemark A, Cant E, Carreto L, et al. A point-of-care neutrophil elastase activity assay identifies bronchiectasis severity, airway infection and risk of exacerbation. Eur Respir J 2019; 53: 1900303 [https://doi.org/10.1183/13993003.00303-2019].

\section{ABSTRACT}

Introduction: Neutrophil elastase activity in sputum can identify patients at high risk of airway infection and exacerbations in bronchiectasis. Application of this biomarker in clinical practice is limited, because no point-of-care test is available. We tested whether a novel semi-quantitative lateral flow device (neutrophil elastase airway test stick - NEATstik ${ }^{\star}$ ) can stratify bronchiectasis patients according to severity, airway infection and exacerbation risk.

Methods: Sputum samples from 124 patients with stable bronchiectasis enrolled in the UK and Spain were tested using the NEATstik ${ }^{\oplus}$, which scores neutrophil elastase concentration from $0\left(<8 \mu \mathrm{g} \cdot \mathrm{mL}^{-1}\right.$ elastase activity) to 10 (maximum detectable neutrophil elastase activity). High neutrophil elastase activity was regarded as a NEATstik ${ }^{\circledast}$ grade $>6$. Severity of disease, airway infection from sputum culture and exacerbations over the 12 months were recorded. An independent validation was conducted in 50 patients from Milan, Italy. Measurements and main results: Patients had a median age of 69 years and forced expiratory volume in $1 \mathrm{~s}$ (FEV1) 69\%. High neutrophil elastase activity was associated with worse bronchiectasis severity using the bronchiectasis severity index $(\mathrm{p}=0.0007)$ and FEV1 $(\mathrm{p}=0.02)$. A high NEATstik ${ }^{\circledast}$ grade was associated with a significant increase in exacerbation frequency, incident rate ratio 2.75 (95\% CI 1.63-4.64, $\mathrm{p}<0.001$ ). The median time to next exacerbation for patients with a NEATstik ${ }^{\bullet}$ grade $>6$ was 103 days compared to 278 days. The hazard ratio was 2.59 (95\% CI 1.71-3.94, p<0.001). Results were confirmed in the independent validation cohort.

Conclusions: A novel lateral flow device provides assessment of neutrophil elastase activity from sputum in minutes and identifies patients at increasing risk of airway infection and future exacerbations. 


\section{Introduction}

Bronchiectasis is a complex clinical syndrome with a wide spectrum of underlying causes [1]. The outcome of bronchiectasis can be unpredictable [2]. Although there are prognostic scores to identify patients at higher risk of mortality, these have limitations such as the inclusion of fixed variables such as age [2-4]. Airway infection is one of the most important "treatable traits" in bronchiectasis because chronic infection with Pseudomonas aeruginosa is linked to a significant increased risk of future mortality, hospital admissions and worse quality of life [5-7]. Infection with other pathogens also provokes airway neutrophilic inflammation and a higher risk of future exacerbations, particularly if bacterial loads are high [8]. Alongside airway infection, exacerbations are critical events in bronchiectasis as they are associated with a significant decrement in quality of life and contribute to disease progression $[9,10]$.

For this reason, the majority of therapies for bronchiectasis recommended by the European Respiratory Society (ERS) guidelines either target the suppression of airway infection, such as long-term macrolides or inhaled antibiotics, or aim to reduce exacerbation frequency $[11,12]$.

A biomarker that could identify patients in real time that are at risk of future exacerbations or have active airway infection would help to identify patients in need for intensified treatment.

Neutrophil elastase activity is a leading candidate for a bronchiectasis biomarker [13]. The relationship between sputum neutrophil elastase activity and airway infection has been known for more than two decades and studies demonstrate that neutrophil elastase may directly impact on bronchiectasis disease progression through effects of ciliated epithelium, mucus production, emphysema development and inactivation of the immune response [14-16]. A systematic review of clinical studies in bronchiectasis identified 31 studies and found that higher neutrophil elastase activity in sputum was associated with sputum colour, $P$. aeruginosa infection, airway bacterial load, disease severity, forced expiratory volume in $1 \mathrm{~s}$ (FEV1) symptoms and long-term risk of exacerbations and lung function decline [16].

Therefore, neutrophil elastase has been tested and validated as a biomarker across multiple studies [13, 16-18]. However, it has not been implemented into clinical practice, in part due to the difficulty of standardising sputum processing and performing ELISA or chromogenic assays in clinical laboratories. A point-of-care lateral flow device that could evaluate neutrophil elastase in minutes would overcome these limitations and might help to stratify patients in terms of disease severity and future risk.

In this study we tested the predictive value of a commercially available point-of-care neutrophil elastase assay (NEATstik ${ }^{\circledR}$ to predict clinically relevant outcomes in patients with bronchiectasis.

\section{Methods}

Patients were enrolled while in a stable phase of their disease from specialist bronchiectasis clinics in Ninewells Hospital (Dundee, UK) and Hospital Sant Pau (Barcelona, Spain) from 2016 to 2017. The inclusion criteria were high-resolution computed tomography confirmed bronchiectasis, the clinical syndrome of bronchiectasis defined by cough, sputum production and/or recurrent respiratory tract infections, and the ability to produce a sputum sample spontaneously at the study visit. Exclusion criteria were known cystic fibrosis, traction bronchiectasis due to interstitial lung disease, age $<18$ years and inability to give informed consent to participate. The study was approved by the local research ethics committees.

\section{Patients and clinical outcomes}

Following informed consent, we collected information about demographics, clinical characteristics, disease burden, previous microbiology and current treatments. Sputum samples were sent for bacterial culture and computed tomography scans were scored using the modified Reiff scale [2]. Patients underwent spirometry according to American Thoracic Society/ERS guidelines. Quality of life was evaluated using the quality of life bronchiectasis respiratory symptoms score. The bronchiectasis severity index was calculated as previously described [2]. Data were collated in the EMBARC case report form including sputum colour measured using the Murray colour chart [19]. Chronic infection was defined as isolation of the same pathogen on two or more occasions $\geqslant 3$ months apart while clinically stable over 1 year.

\section{Sputum processing and neutrophil elastase measurement}

Neutrophil elastase was measured using two methods: a validated activity-based immunoassay and the point-of-care "NEATstik ${ }^{\circledast "}$ " test. For immunoassay, spontaneously expectorated samples were diluted with $8 \times$ volume phosphate buffered saline and centrifuged at $3000 \times \mathrm{g}$ for $15 \mathrm{~min}$ and the supernatant carefully removed, as previously described [13]. Dithiothreitol was not used. Samples were diluted and neutrophil elastase activity measured according to the manufacturer's instructions. For the NEATstik method, spontaneously expectorated sputum samples were diluted immediately $10 \times$ in buffer provided in the kit 
FIGURE 1 NEATstik ${ }^{\circledR}$ scoring system. A value of $0-10$ is awarded, based on the intensity of the band.

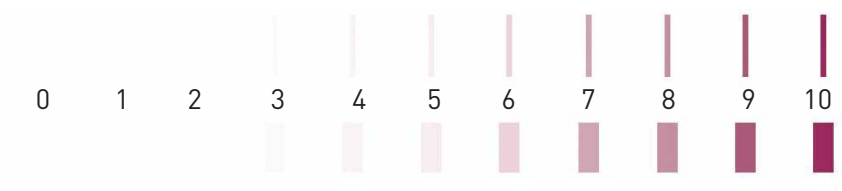

and inverted 10 times. The diluted sample was then added to the NEATstik ${ }^{\otimes}$ lateral flow device. The test consists of a control line and a test line. According to the manufacturer's instructions the intensity of the test line corresponds to the neutrophil elastase concentration on a 10-point scale (figure 1), and a positive test corresponds to an approximate concentration of $8 \mu \mathrm{g} \cdot \mathrm{mL}^{-1}$. A video showing the testing procedure is included in the supplementary material.

\section{Exacerbation study}

A subset of patients were asked to contact the study site when they experienced an exacerbation and to provide a spontaneous sputum sample. In this convenience sample, patients were reviewed by a clinician and exacerbation defined according to the British Thoracic Society definition [20]. Bacterial exacerbations were defined by the presence of a cultured pathogen and viral exacerbations defined by the presence of a positive PCR.

\section{Validation}

The reliability and reproducibility of the NEATstik ${ }^{\circledast}$ scoring system was tested. Each processed sputum sample was scored by two blinded independent observers. Observer agreement was evaluated using BlandAltman plots, linear regression and the area under the receiver operator characteristic curve. Image analysis was conducted to provide a third validation and proof of concept that digital validation of photographs taken on a mobile phone camera could be used for future development of an app or similar assessment device. A subset of 50 images were photographed, converted to an 8-bit greyscale image and adjusted to obtain a consistent background and control line intensity. Mean pixel intensity of the NEATstik $^{\infty}$ test band was quantified in FIJI (Image); opensource software, National Institutes of Health, Bethesda, MD, USA).

Additionally, agreement between the NEATstik ${ }^{\circledR}$ assay and the immunoassay was tested.

\section{Validation cohort}

An independent validation study was performed at a specialist bronchiectasis clinic (Policlinico Hospital, Milan, Italy). The NEATstik ${ }^{\oplus}$ assay was performed on fresh sputum samples at the point of care in 50 patients; 25 with chronic bacterial infection and 25 without chronic infection. Clinical data were collected, but no follow-up data were collected in this cohort. NEATstik ${ }^{\bullet}$ results were verified by two observers.

\section{Statistical analysis}

Analysis was performed double-blind, with staff performing the scoring of the NEATstik ${ }^{\circledast}$ grade blinded to patient characteristics and the staff who obtained sputum samples and collated clinical data blinded to the results of the NEATstik ${ }^{\circledR}$ test until the end of the study. For correlating neutrophil elastase activity with clinical parameters and long-term outcomes, two cut-offs were used. First, a comparison between samples testing positive (any grade 1-10) versus negative; and second, a comparison between the highest

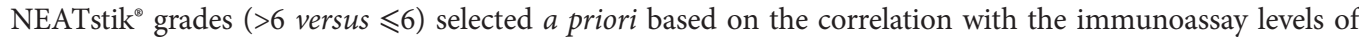
neutrophil elastase.

Analysis was performed using SPSS 22.0. Statistical differences among continuous variables were analysed using t-test and ANOVA or the appropriate nonparametrical test. Frequency of exacerbations and hospital admissions during the study were analysed using a negative binomial model with follow-up time as an offset. Time to first exacerbation was evaluated using Kaplan-Meier survival analysis. We defined statistical significance as a two-tailed $\mathrm{p}<0.05$.

\section{Results}

\section{Patient characteristics}

124 patients were included in the study. The patient characteristics summarised in table 1 were consistent with previously reported characteristics of European bronchiectasis populations.

\section{Accuracy and reproducibility of neutrophil elastase measurement}

There was a clear and statistically significant correlation between neutrophil elastase activity measured using the immunoassay and the NEATstik ${ }^{\oplus}$ result (Spearman correlation $\mathrm{r}=0.73, \mathrm{p}<0.001$ ). 


\begin{tabular}{|c|c|}
\hline \multicolumn{2}{|l|}{ Demographics } \\
\hline Age years & $69(62-78)$ \\
\hline Female & $71(57.3)$ \\
\hline BMI $\mathrm{kg} \cdot \mathrm{m}^{-2}$ & $25.6(22.4-29.8)$ \\
\hline Ex-smokers & $50(40.3)$ \\
\hline Current smokers & 9 (7.3) \\
\hline \multicolumn{2}{|l|}{ Aetiology } \\
\hline Idiopathic & $64(51.6)$ \\
\hline Post-infective & $11(8.9)$ \\
\hline COPD & $9(7.3)$ \\
\hline Post-TB & $7(5.6)$ \\
\hline Rheumatoid arthritis & $7(5.6)$ \\
\hline Inflammatory bowel disease & $6(4.8)$ \\
\hline ABPA & $3(2.4)$ \\
\hline Asthma & $3(2.4)$ \\
\hline Immunodeficiency & $3(2.4)$ \\
\hline Other ${ }^{\#}$ & $11(8.9)$ \\
\hline \multicolumn{2}{|l|}{ Disease severity } \\
\hline BSI score & $7(5-11)$ \\
\hline \multicolumn{2}{|l|}{ BSI score risk class } \\
\hline Mild & $31(25)$ \\
\hline Moderate & $44(35.5)$ \\
\hline Severe & 49 (39.5) \\
\hline \multicolumn{2}{|l|}{ Clinical status } \\
\hline Sputum volume $\mathrm{mL} \cdot$ day $^{-1}$ & $15(5-30)$ \\
\hline MRC dyspnoea score & $2(1-2)$ \\
\hline Exacerbations in the previous year & $2(1-4)$ \\
\hline At least one hospitalisation in the previous year & $37(29.8)$ \\
\hline \multicolumn{2}{|l|}{ Functional status } \\
\hline FEV1 \% predicted & $67.9(50.9-81.5)$ \\
\hline \multicolumn{2}{|l|}{ Microbiology } \\
\hline$P$. aeruginosa chronic infection & $35(28.2)$ \\
\hline Negative sputum culture & $62(50.0)$ \\
\hline \multicolumn{2}{|l|}{ Treatment } \\
\hline Long-term macrolide & $51(41.1)$ \\
\hline Inhaled antibiotic & 18 (14.5) \\
\hline \multicolumn{2}{|l|}{ Quality of life } \\
\hline Quality of life bronchiectasis questionnaire respiratory symptom score & $59.3(46.8-77.8)$ \\
\hline \multicolumn{2}{|c|}{$\begin{array}{l}\text { Data are presented as median (interquartile range) or } \mathrm{n}(\%) \text {. BMI: body mass index; COPD: chronic } \\
\text { obstructive pulmonary disease; TB: tuberculosis; ABPA: allergic bronchopulmonary aspergillosis; BSI: } \\
\text { bronchiectasis severity index; MRC: Medical Research Council; FEV1: forced expiratory volume in } 1 \mathrm{~s} ; \\
\text { P. aeruginosa: Pseudomonas aeruginosa. }{ }^{\#} \text { : included } \alpha_{1} \text {-antitrypsin deficiency, obstruction, primary ciliary } \\
\text { dyskinesia and tracheobronchomegaly. }\end{array}$} \\
\hline
\end{tabular}

The two blinded observers showed a high level of agreement $(\mathrm{r}=0.93, \mathrm{p}<0.001)$. There was a strong agreement between both observers and quantification of the test line by image analysis of digital photographs $(\mathrm{n}=50$; observer $1 \mathrm{r}=0.81, \mathrm{p}<0.001$ and observer $2 \mathrm{r}=0.91, \mathrm{p}<0.001) .21$ sputum samples had no detectable neutrophil elastase activity (grade 0$)$. There was agreement on 20 (95.2\%) out of 21 negative samples between observers (one awarded grade 1 for a single sample that the original observer rated as negative). Based on the low number of negative results, we set a cut-off value of $>6$ as a positive or "high-risk" test. The $\kappa$ statistic for agreement using this cut-off was 0.82 (0.73-0.91). The Bland-Altman plot is shown in supplementary figure E1. We conclude that scoring can be performed with a high degree of reproducibility.

\section{Relationship between point-of-care neutrophil elastase assay and cross-sectional severity of} disease

Compared to patients without chronic infection, patients with $P$. aeruginosa had significantly higher neutrophil elastase activity (figure $2 a)$, as did patients with other chronic infections $(p=0.004)$. Of those without detectable neutrophil elastase activity, only one had chronic infection. The sensitivity and specificity for detection of chronic infection were $71.9 \%$ and $55.1 \%$, respectively, for a NEATstik grade $^{\circledR} 6$, 

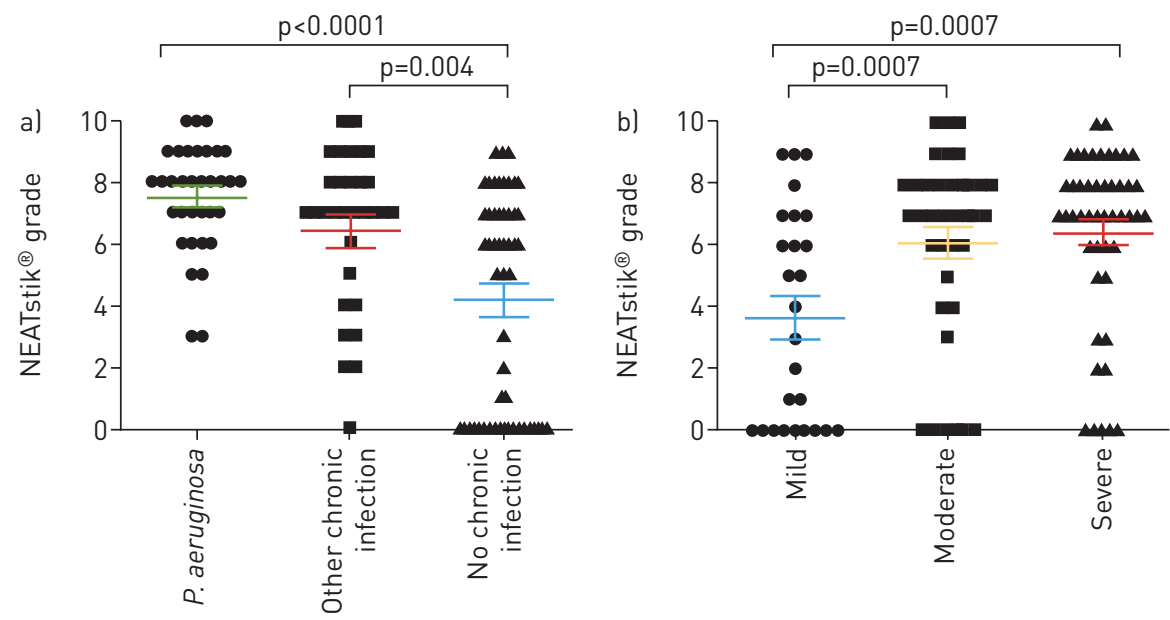

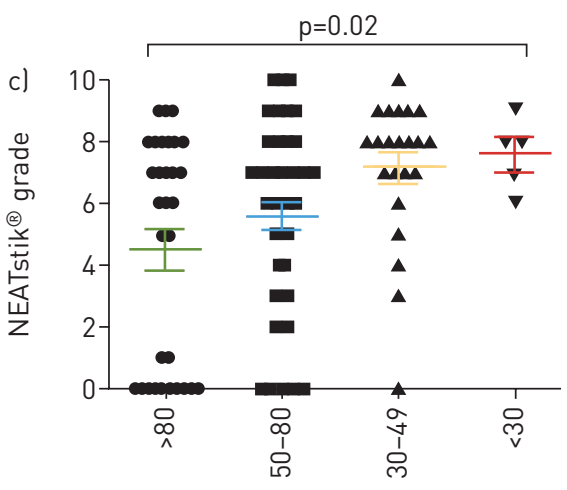

FEV $1 \%$ predicted

FIGURE 2 The relationship between the NEATstik ${ }^{\circledR}$ grade, airway infection and severity of disease. a) Airway infection, b) bronchiectasis severity index, c) forced expiratory volume in $1 \mathrm{~s}\left(\mathrm{FEV}_{1}\right)$ groups. Data are shown as mean \pm SEM. Coloured error bars are to improve visualisation rather than having any significance.

and $98.3 \%$ and $31.3 \%$ for positive versus negative NEATstik ${ }^{\circledR}$ results. The corresponding sensitivity and specificity for detecting $P$. aeruginosa infection were $100 \%$ and $23.6 \%$, respectively.

Figure $2 \mathrm{~b}$ shows the relationship between neutrophil elastase and the bronchiectasis severity index (BSI). Patients with more severe bronchiectasis had higher neutrophil elastase activity $(\mathrm{p}<0.001)$. The linear correlation between neutrophil elastase grade and BSI was $\mathrm{r}=0.31(0.14-0.47, \mathrm{p}<0.001)$. Neutrophil elastase grade was also correlated with Medical Research Council MRC dyspnoea score ( $r=0.25$, 95\% CI 0.07-0.42; $\mathrm{p}=0.008)$, FEV1 $(\mathrm{r}=-0.33,95 \%$ CI $-0.49--0.16 ; \mathrm{p}<0.001)$ and the quality-of-life bronchiectasis questionnaire respiratory symptom score $(\mathrm{r}=-0.37,95 \% \mathrm{CI}-0.56--0.15 ; \mathrm{p}=0.002)$. Correlations, including those with bacterial infection, BSI and FEV1 were all consistent when evaluated individually in both the Spanish and UK cohorts.

Classifying NEATstik ${ }^{\otimes}$ results as simply positive and negative showed relationships with clinical parameters, with positive tests being associated with a higher BSI score, lower FEV1 and the presence of airway infection (supplementary figure E2).

\section{Predictive value for future exacerbations}

A high NEATstik ${ }^{\otimes}$ grade $(>6)$ was predictive of future exacerbations. The median time to next exacerbation for patients with a NEATstik ${ }^{\circledast}$ grade $>6$ was 103 days, compared to 278 days for those with lower NEATstik $^{\oplus}$ grades. The Kaplan-Meier survival curve is shown in figure 3a. The hazard ratio was
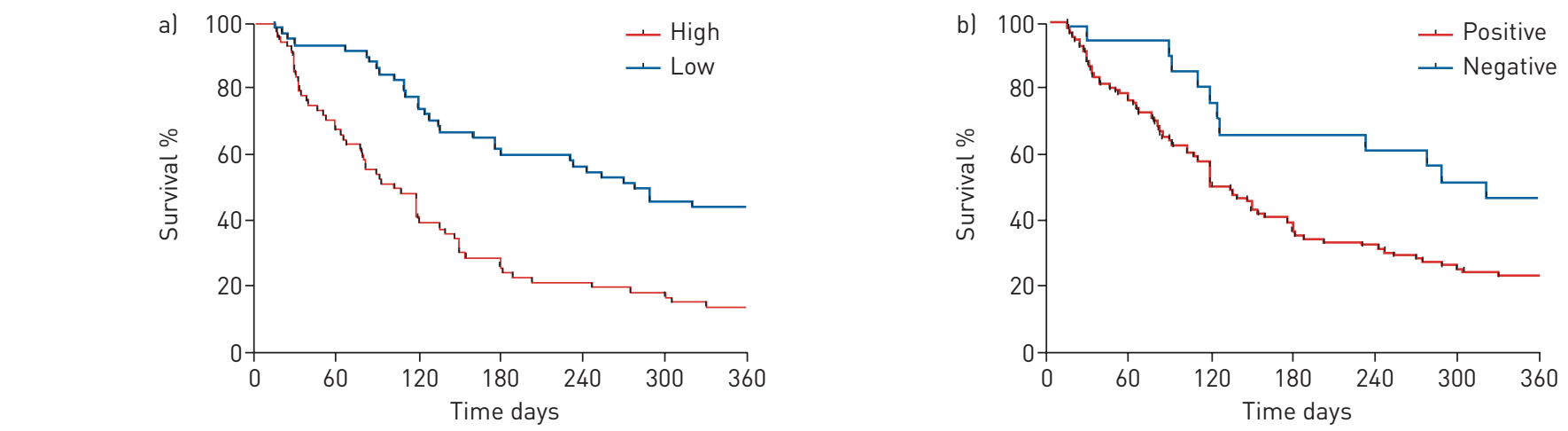

At risk $\mathrm{n}$

High

$\begin{array}{llllllll}64 & 47 & 31 & 19 & 15 & 12 & 0 & \text { Positive } \\ 57 & 54 & 44 & 35 & 33 & 28 & 0 & \text { Negative }\end{array}$

At risk $n$

Positive

Low

$\begin{array}{ccccccc}103 & 80 & 58 & 40 & 34 & 27 & 0 \\ 21 & 21 & 17 & 15 & 14 & 12 & 0\end{array}$

FIGURE 3 Time to first exacerbation according to baseline NEATstik ${ }^{\circledR}$ neutrophil elastase result. a) High (NEATstik ${ }^{\circledR}$ grade $>6$ ) versus low scores; b) positive (any grade $>0$ ) versus negative result. 
2.59 (95\% CI $1.71-3.94, \mathrm{p}<0.001)$. Similarly, comparing positive versus negative results, there was a statistically significant difference in time to the next exacerbation event. The median time was 135 days for patients with a positive test versus 320 days for patients with a negative test. The hazard ratio was 2.15 (95\% CI 1.32-3.51, $\mathrm{p}=0.01)$.

A high NEATstik ${ }^{\circledast}$ level was associated with a significant increase in exacerbation frequency during follow-up with an incident rate ratio of 2.75 (95\% CI 1.63-4.64, p<0.001) from the negative binomial model. There was consistency between the UK and Spain cohorts and in terms of prediction of exacerbations (supplementary figure E3).

Severe exacerbations requiring hospitalisation were infrequent during this study, occurring in 20 patients (mean \pm SD $0.29 \pm 0.75$ per year). 19 out of 20 patients requiring hospitalisation had an elevated NEATstik ${ }^{\circ}$ grade $>6$. The single patient with a level $<6$ had a negative NEATstik ${ }^{\circledR}$. The calculated risk ratio for a NEATstik $^{\circledast}$ grade $>6$ was 12.8 (95\% CI 1.77-91.9, $\left.\mathrm{p}<0.001\right)$. The equivalent for positive versus negative NEATstik $^{\oplus}$ results was 3.87 (95\% CI $0.55-27.4, \mathrm{p}=0.2$ ). It should be noted that 12 out of 20 patients requiring hospitalisation had chronic $P$. aeruginosa infection, which we had already observed to be associated with high neutrophil elastase activity in sputum.

\section{Neutrophil elastase activity during exacerbations}

42 patients attended for visits during protocol-defined exacerbations. Details of sputum culture results at exacerbation are shown in the supplementary material. As previously observed with neutrophil elastase ELISA [21], changes in neutrophil elastase at exacerbation were heterogeneous and NEATstik ${ }^{\circledR}$ was not useful in differentiating stable state from exacerbation.

Bacterial exacerbations had higher mean NEATstik ${ }^{\star}$ grades, as shown in figure 4 . Comparisons with both viral and negative exacerbations were statistically significant ( $\mathrm{p}=0.005$ and $\mathrm{p}=0.001$, respectively). The small numbers did not allow a meaningful comparison between viral and "negative" exacerbations.

\section{Independent validation}

The characteristics of the validation cohort are shown in supplementary table E2. The relationship between NEATstik $^{\oplus}$ grade and chronic infection was confirmed in this cohort (figure 5a and b). There was a strong agreement between two independent observers for NEATstik ${ }^{\star}$ grade, with the correlation for two physicians $(\mathrm{r}=0.91,95 \% \mathrm{CI} 0.84-0.95 ; \mathrm{p}<0.001)$ and for scoring performed by a nurse $(\mathrm{r}=0.91,95 \% \mathrm{CI}$ $0.84-0.95 ; \mathrm{p}<0.001)$ and by a physiotherapist $(\mathrm{r}=0.92,95 \%$ CI $0.86-0.95 ; \mathrm{p}<0.001)$ showing similar reproducibility. NEATstik ${ }^{\oplus}$ result was not significantly correlated with the sputum colour chart $(r=0.18$, $\mathrm{p}=0.2)$ but was correlated with sputum volume $(\mathrm{r}=0.39,95 \%$ CI $0.10-0.62 ; \mathrm{p}=0.01)$. Sputum colour was not associated with BSI $(\mathrm{p}=0.06)$ or chronic infection $(\mathrm{p}=0.1)$.

\section{Discussion}

To the best of the authors' knowledge, this is the first description of a rapid point-of-care test that can identify the presence of airway infection and the risk of future exacerbations in patients with bronchiectasis. The test is semi-quantitative and shows a good correlation with neutrophil elastase activity measured by immunoassay. Neutrophil elastase activity is an established biomarker of bronchiectasis as

FIGURE 4 Neutrophil elastase NEATstik ${ }^{\circledR}$ results at exacerbation in 42 patients: bacterial exacerbations $(n=25)$, viral exacerbations $(n=8)$ and exacerbations where both sputum culture and viral PCR were negative $(\mathrm{n}=7)$. Data are presented as mean \pm SEM.

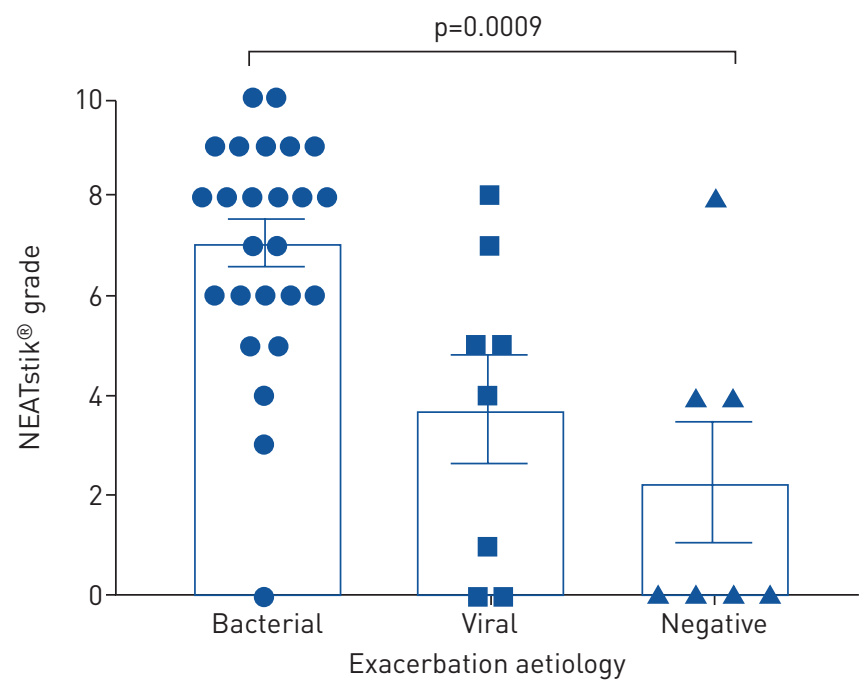



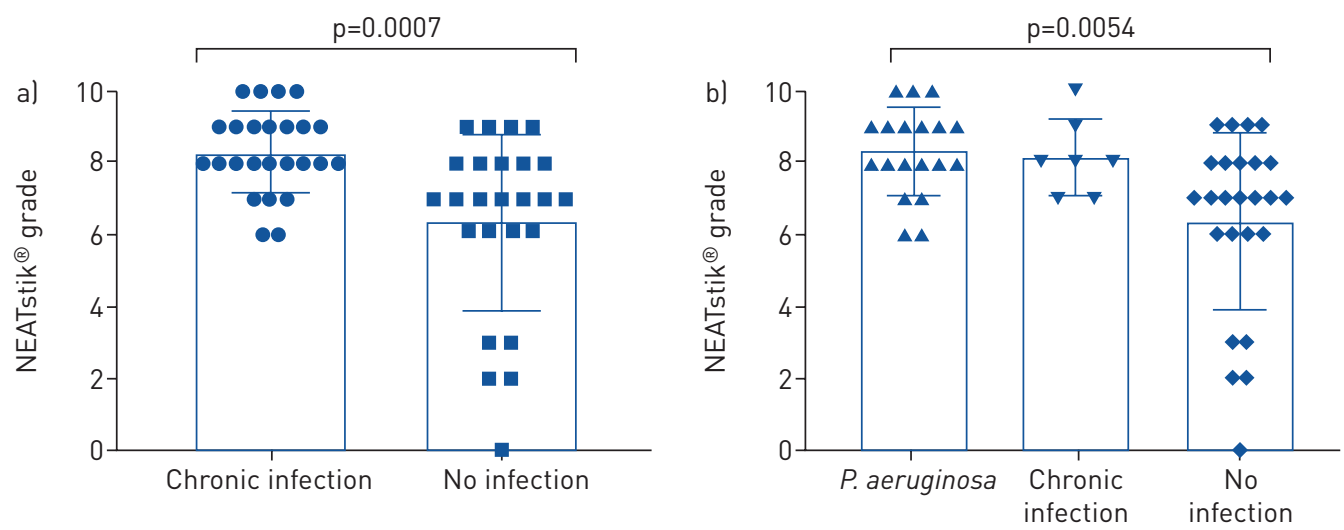

FIGURE 5 Chronic infection status and NEATstik ${ }^{\circledR}$ grade. a) All chronic infection, including Pseudomonas aeruginosa and other pathogens; b) separating $P$. aeruginosa and other pathogens.

well as being associated with risk of exacerbations, airway infection status and lung function decline in other conditions such as cystic fibrosis [16]. Implementation of sputum biomarkers into clinical practice are limited by the requirement to process sputum via centrifugation and the design of traditional assays for neutrophil elastase, which are typically based on the cleavage of chromogenic substrates. Therefore, the NEATstik ${ }^{\circ}$ method has a number of advantages over existing methods of neutrophil elastase quantification. The procedure requires only a sample container, a weighing balance and the NEATstik ${ }^{\circ}$ device and a result can be obtained within minutes. It is feasible to perform this test in a clinic setting or for a patient to perform the test at home.

Neutrophil elastase is released from azurophilic granules of neutrophils during degranulation or neutrophil extracellular trap formation [22]. The strongest driver of neutrophil recruitment to the airway and subsequent degranulation is bacterial infection [8]. Several studies have shown that patients with $P$. aeruginosa infection have high levels of neutrophil elastase activity $[8,13,17,18]$. Previous work shows a strong relationship between neutrophil elastase activity and bacterial load, but for pragmatic reasons we did not perform quantitative bacteriology in this study. Patients with more severe bronchiectasis had higher neutrophil elastase activity in this study, as previously reported [13]. The BSI is a composite score that includes age, body mass index, MRC dyspnoea score, radiological bronchiectasis, exacerbations, hospitalisations, FEV1 and chronic infection. We show here that the relationship between neutrophil elastase activity and severity is driven by worse symptoms of breathlessness, more exacerbations, worse lung function and more chronic infection in patients with higher levels of neutrophil elastase.

We identified very few samples (21 out of 124 in stable patients) that were truly negative using the NEATstik $^{\circ}$ assay. For this reason, the semi-quantitative scoring may be most useful in clinical practice, since a positive/negative approach may be too sensitive and insufficiently specific. Conversely, the $100 \%$ sensitivity we observed for $P$. aeruginosa infection suggests that, if validated in further independent cohorts, a negative test could be used to effectively exclude the presence of $P$. aeruginosa infection without requiring culture. We found that the semi-quantitative grading could be performed reliably and reproducibly between independent observers.

Exacerbations are key drivers of disease progression in bronchiectasis. Elevated neutrophil elastase predicted a shorter time to the next exacerbation and a higher frequency of exacerbations during follow-up. The magnitude of this effect with the NEATstik ${ }^{\circ}$ method was similar to that previously observed with an immunoassay in an independent cohort [13]. The prediction of exacerbations to guide preventative therapy is a key unmet need in the management of bronchiectasis patients. The ERS guidelines recommend long-term macrolides, long-term inhaled antibiotics and some mucoactive therapies to patients at risk of future exacerbations, but at present the only method available to predict future exacerbations is the history of previous exacerbations [9]. While these are strongly correlated, recent experience suggests that this method is not always reliable. In the RESPIRE trials of inhaled dry powder ciprofloxacin, the inclusion criteria required at least two exacerbations in the previous year [23-25]. The subsequent rates of exacerbation of $0.6-1$ per year in the placebo groups of these trials suggest a failure to enrich for patients at high risk of exacerbation events. Therefore, a point-of-care test that can identify patients at risk would be useful in clinical practice to guide therapy and to enrich clinical trials.

We performed a pilot study of 42 exacerbations to investigate if different exacerbation types had different levels of neutrophil elastase. We found a clear association between elevated neutrophil elastase positive 
bacterial culture at exacerbation. It is important to note that negative NEATstik ${ }^{\circledast}$ tests were observed at exacerbation, confirming that neutrophil elastase cannot be used to diagnose exacerbation, since there appear to be phenotypes of exacerbation that are not associated with elevated neutrophilic inflammation [13]. Nevertheless, our initial observation would suggest the future potential to guide antibiotic treatment of exacerbations based on the NEATstik ${ }^{\oplus}$ result.

Our study has important limitations. The sample size was sufficient to demonstrate statistically significant differences between the groups, but given the heterogeneity of bronchiectasis it would be important to investigate the NEATstik ${ }^{\circledR}$ assay in a larger cohort of patients. Our study was limited to 12 months of follow-up and there were relatively few severe exacerbations on which to base any strong conclusions regarding this end-point. As the participating hospitals are tertiary referral centres, the study included a high proportion of patients with severe bronchiectasis, and therefore care should be taken if extrapolating results to a milder population. Our independent validation cohort included only 50 patients and was designed to validate the association with airway infection; it was not powered to test the association with exacerbations. Further large studies are still needed. An important limitation of our respiratory infection data is the reliance on traditional culture methods. Studies are increasingly using more sensitive methods such as quantitative PCR or sequencing of the airway microbiome to define airway infection [26]. These methods are not yet implemented in clinical practice and so we regard culture as an appropriate gold standard for our analysis, albeit one with important limitations. Despite these limitations, our study has many strengths, including the extensive validation of the assay, the multicentre design with consistent results between Spain, Italy and the UK and the comprehensive clinical data recorded.

In summary, a simple point-of-care semi-quantitative neutrophil elastase assay can identify bronchiectasis patients with airway infection and patients at high risk of exacerbations over the subsequent 12 months. Future studies should evaluate whether implementation of such an assay in clinical practice or clinical trials can be used to improve risk stratification or therapeutic decision making.

Author contributions: Study design: A. Shoemark, S. Aliberti, O Sibila and J.D. Chalmers. Patient recruitment and data collection: A. Shoemark, L. Carreto, M. Oriano, L. Perea, E. Canto, L. Terranova, S. Vidal, S. Aliberti, O Sibila and J.D. Chalmers. Laboratory assays: A. Shoemark, E. Cant, L. Carreto, A. Smith, M. Oriano, H.R. Keir, L. Terranova and K. Moffitt. Data analysis: E. Cant, L. Carreto, S. Aliberti and J.D. Chalmers. Writing of the manuscript: A. Shoemark, O Sibila and J.D. Chalmers. Revising of the manuscript and approval of submission: all authors.

Conflict of interest: A. Shoemark has nothing to disclose. E. Cant has nothing to disclose. L. Carreto has nothing to disclose. A. Smith has nothing to disclose. M. Oriano has nothing to disclose. H.R. Keir has nothing to disclose. L. Perea has nothing to disclose. E. Canto has nothing to disclose. L. Terranova has nothing to disclose. S. Vidal has nothing to disclose. K. Moffitt was employed by Proaxsis, during the conduct of the study. S. Aliberti has nothing to disclose. O Sibila has nothing to disclose. J.D. Chalmers reports grants and personal fees from GlaxoSmithKline, Boehringer Ingelheim, Pfizer, Bayer Healthcare, Grifols and Insmed, grants from AstraZeneca, personal fees from Napp and Aradigm Corporation, outside the submitted work.

Support statement: This study was funded by the European Respiratory Society through the EMBARC2 consortium. EMBARC2 is supported by project partners Chiesi, Grifols, Insmed, Novartis and Zambon. J.D. Chalmers is supported by the GSK/British Lung Foundation Chair of Respiratory Research. O. Sibila is supported by PERIS 2017. The neutrophil elastase assay was provided by Proaxsis Ltd. Funding information for this article has been deposited with the Crossref Funder Registry.

\section{References}

1 Shoemark A, Ozerovitch L, Wilson R. Aetiology in adult patients with bronchiectasis. Respir Med 2007; 101: $1163-1170$

2 McDonnell MJ, Aliberti S, Goeminne PC, et al. Multidimensional severity assessment in bronchiectasis: an analysis of seven European cohorts. Thorax 2016; 71: 1110-1118.

3 Ellis HC, Cowman S, Fernandes M, et al. Predicting mortality in bronchiectasis using bronchiectasis severity index and FACED scores: a 19-year cohort study. Eur Respir J 2016; 47: 482-489.

4 Martínez-García MA, de Gracia J, Vendrell Relat M, et al. Multidimensional approach to non-cystic fibrosis bronchiectasis: the FACED score. Eur Respir J 2014; 43: 1357-1367.

5 Boaventura R, Sibila O, Agusti A, et al. Treatable traits in bronchiectasis. Eur Respir J 2018; 52: 1801269.

6 Finch S, McDonnell MJ, Abo-Leyah H, et al. A comprehensive analysis of the impact of Pseudomonas aeruginosa colonization on prognosis in adult bronchiectasis. Ann Am Thorac Soc 2015; 12: 1602-1611.

7 Araújo D, Shteinberg M, Aliberti S, et al. The independent contribution of Pseudomonas aeruginosa infection to long-term clinical outcomes in bronchiectasis. Eur Respir J 2018; 51: 1701953.

8 Chalmers JD, Smith MP, McHugh BJ, et al. Short- and long-term antibiotic treatment reduces airway and systemic inflammation in non-cystic fibrosis bronchiectasis. Am J Respir Crit Care Med 2012; 186: 657-665.

9 Chalmers JD, Aliberti S, Filonenko A, et al. Characterization of the "frequent exacerbator phenotype" in bronchiectasis. Am J Respir Crit Care Med 2018; 197: 1410-1420.

10 Gao Y-H, Guan W-J, Xu G, et al. The role of viral infection in pulmonary exacerbations of bronchiectasis in adults: a prospective study. Chest 2015; 147: 1635-1643. 
11 Polverino E, Goeminne PC, McDonnell MJ, et al. European Respiratory Society guidelines for the management of adult bronchiectasis. Eur Respir J 2017; 50: 1700629.

12 Hill AT, Haworth CS, Aliberti S, et al. Pulmonary exacerbation in adults with bronchiectasis: a consensus definition for clinical research. Eur Respir J 2017; 49: 1700051.

13 Chalmers JD, Moffitt KL, Suarez-Cuartin G, et al. Neutrophil elastase activity is associated with exacerbations and lung function decline in bronchiectasis. Am J Respir Crit Care Med 2017; 195: 1384-1393.

14 Gehrig S, Duerr J, Weitnauer M, et al. Lack of neutrophil elastase reduces inflammation, mucus hypersecretion, and emphysema, but not mucus obstruction, in mice with cystic fibrosis-like lung disease. Am J Respir Crit Care Med 2014; 189: 1082-1092.

15 Amitani R, Wilson R, Rutman A, et al. Effects of human neutrophil elastase and Pseudomonas aeruginosa proteinases on human respiratory epithelium. Am J Respir Cell Mol Biol 1991; 4: 26-32.

16 Gramegna A, Amati F, Terranova L, et al. Neutrophil elastase in bronchiectasis. Respir Res 2017; 18: 211.

17 Tsang KW, Chan K, Ho P, et al. Sputum elastase in steady-state bronchiectasis. Chest 2000; 117: 420-426.

18 Goeminne PC, Vandooren J, Moelants EA, et al. The Sputum Colour Chart as a predictor of lung inflammation, proteolysis and damage in non-cystic fibrosis bronchiectasis: a case-control analysis. Respirology 2014; 19: 203-210.

19 Chalmers JD, Aliberti S, Polverino E, et al. The EMBARC European Bronchiectasis Registry: protocol for an international observational study. ERJ Open Res 2016; 2: 00081-2015.

20 Pasteur MC, Bilton D, Hill AT. British Thoracic Society guideline for non-CF bronchiectasis. Thorax 2010; 65: 577.

21 Chalmers JD, Chotirmall SH. Bronchiectasis: new therapies and new perspectives. Lancet Respir Med 2018; 6: 715-726.

22 Stockley JA, Walton GM, Lord JM, et al. Aberrant neutrophil functions in stable chronic obstructive pulmonary disease: the neutrophil as an immunotherapeutic target. Int Immunopharmacol 2013; 17: 1211-1217.

23 De Soyza A, Aksamit T, Bandel T-J, et al. RESPIRE 1: a phase III placebo-controlled randomised trial of ciprofloxacin dry powder for inhalation in non-cystic fibrosis bronchiectasis. Eur Respir J 2018; 51: 1702052.

24 Aksamit T, De Soyza A, Bandel T-J, et al. RESPIRE 2: a phase III placebo-controlled randomised trial of ciprofloxacin dry powder for inhalation in non-cystic fibrosis bronchiectasis. Eur Respir J 2018; 51: 1702053.

25 Chotirmall SH, Chalmers JD. RESPIRE: breathing new life into bronchiectasis. Eur Respir J 2018; 51: 1702444.

26 Faner R, Sibila O, Agustí A, et al. The microbiome in respiratory medicine: current challenges and future perspectives. Eur Respir J 2017; 49: 1602086. 